\title{
Three-point boundary value problems in Banach spaces
}

\author{
Dionicio Pastor Dallos Santos* \\ Department of Mathematics, IME-USP, Cidade Universitária, \\ CEP 05508-090, São Paulo, SP, Brazil
}

\begin{abstract}
Using degree for $\alpha$-condensing maps, we obtain the existence of at least one solution for nonlinear boundary value problems$$
\left\{\begin{array}{l}
\left(\varphi\left(u^{\prime}\right)\right)^{\prime}=f\left(t, u, u^{\prime}\right) \\
u(0)=u(1)=u^{\prime}(0)
\end{array}\right.
$$

where $\varphi: X \rightarrow X$ is a linear homeomorphism, $f:[0,1] \times X \times X \rightarrow X$ is a continuous function and $X$ is a real Banach space.
\end{abstract}

Mathematics Subject Classification (2010). 34B15; 47H08; 47H11.

Key words: boundary value problems, degree for $\alpha$-condensing maps, measure of noncompactness.

The author declares that there is no conflict of interest regarding the publication of this article.

\section{Introduction}

The purpose of this article is to obtain some existence results for the nonlinear boundary value problem of the form

$$
\left\{\begin{array}{l}
\left(\varphi\left(u^{\prime}\right)\right)^{\prime}=f\left(t, u, u^{\prime}\right) \\
u(0)=u(1)=u^{\prime}(0)
\end{array}\right.
$$

where $\varphi: X \rightarrow X$ is a linear homeomorphism, $f:[0,1] \times X \times X \rightarrow X$ is a continuous function and $X$ is a real Banach space. We call solution of this problem any function $u:[0,1] \rightarrow X$ of class $C^{1}$ such that the function $t \mapsto \varphi\left(u^{\prime}(t)\right)$ is continuously

${ }^{*}$ Email: dionicio@ime.usp.br 
differentiable, satisfying the boundary conditions and $\left(\varphi\left(u^{\prime}(t)\right)\right)^{\prime}=f\left(t, u(t), u^{\prime}(t)\right)$ for all $t \in[0,1]$.

The existence of solutions for second-order boundary value problems has been studied by many authors using various methods (see [3, 4, 1, 9, 10, 11, 12] and references therein).

In particular, in 10 have studied the following boundary value problem:

$$
\left\{\begin{array}{l}
\left(\varphi\left(u^{\prime}\right)\right)^{\prime}=f\left(t, u, u^{\prime}\right) \\
u(T)=0=u^{\prime}(0)
\end{array}\right.
$$

where $\varphi: X \rightarrow X$ is a homeomorphism with reverse Lipschitz constant such that $\varphi(0)=0, f:[0, T] \times X \times X \rightarrow X$ is a continuous function, $T$ is a positive real number, and $X$ is a real Banach space. They obtained the existence of solutions of (1.2) using Leray-Schauder degree or degree for $\alpha$-condensing maps.

Recently, W.-X. Zhou and J. Peng [12] have studied the following boundary value problem:

$$
\left\{\begin{array}{l}
-u^{\prime \prime}=f(t, u) \\
u(0)=0=u(1)
\end{array}\right.
$$

where $f:[0,1] \times X \rightarrow X$ is a continuous function and $X$ is a Banach space. They obtained the existence of solutions of $(1.3)$, where the main tools used in the study are Sadovskii fixed point theorem and precise computation of measure of noncompactess.

Motivated by these results, the main aim of this paper is to investigate the existence of at least one solution for the boundary value problem (1.1) using degree for $\alpha$-condensing maps. For this, we reduce the nonlinear boundary value problem to some fixed points problem. Next, we shall essentially consider one type of regularity assumption for $f(t, x, y)$ expressed in terms of the measure of noncompactness, which allows us to apply the methods of topological degree theory for $\alpha$-condensing maps. The main new features presented in this paper are as follows: First, the extension of some results above cited to a more general type of boundary conditions. Second, the main tool used in the analysis is the degree for $\alpha$-condensing maps. Such problems do not seem to have been studied in the literature.

The rest of the paper is organized as follows. In Section 2, we establish the notation, terminology, and various lemmas which will be used throughout this paper. In section 3, we formulate the fixed point problem equivalent to the problem (1.1). In Section 4, we give main results in this paper. 


\section{Notations and preliminary results}

As usual, $C=C([0,1], X)$ is the Banach space of all continuous functions from $[0,1]$ into $X$ endowed with the norm $\|\cdot\|_{\infty}$, and $C^{1}=C^{1}([0,1], X)$ denote the Banach space of continuously differentiable functions from $[0,1]$ into $X$ equipped with the usual norm $\|u\|_{1}=\max \left\{\|u\|_{\infty},\left\|u^{\prime}\right\|_{\infty}\right\}$.

We introduce the following applications:

the Nemytskii operator $N_{f}: C^{1} \rightarrow C$,

$$
N_{f}(u)(t)=f\left(t, u(t), u^{\prime}(t)\right),
$$

the integration operator $H: C \rightarrow C^{1}$,

$$
H(u)(t)=\int_{0}^{t} u(s) d s .
$$

Throughout this paper, we denote by $(X,\|\cdot\|)$ a real Banach space and $I=[0,1]$. For $A \subseteq C^{1}$, we use the notation;

$$
\begin{gathered}
A(t)=\{u(t): u \in A\}, \\
A(I)=\{u(t): u \in A, t \in I\}, \\
A^{\prime}(t)=\left\{u^{\prime}(t): u \in A\right\}, \\
A^{\prime}=\left\{u^{\prime}: u \in A\right\}, \\
A^{\prime}(I)=\left\{u^{\prime}(t): u \in A, t \in I\right\} .
\end{gathered}
$$

Definition 2.1. Let $X$ be a Banach space and let $M_{X}$ be the family of bounded subsets of $X$. The Kuratowski measure of noncompactness is the map $\alpha: M_{X} \rightarrow$ $[0, \infty)$ defined by

$\alpha(B)=\inf [d>0: B$ admits a finite cover by sets of diameter $\leq d]$; here $B \in M_{X}$.

\section{Properties:}

(a) $\alpha(B)=0$ iff $\bar{B}$ is compact.

(b) $S \subset B$ then $\alpha(S) \leq \alpha(B)$.

(c) $\alpha(\bar{B})=\alpha(B)$.

(d) $\alpha(B \cup S)=\max \{\alpha(B), \alpha(S)\}$.

(e) $\alpha(\lambda B)=|\lambda| \alpha(B)$, where $\lambda \in \mathbb{R}$ and $\lambda B=\{\lambda b: b \in B\}$.

(f) $\alpha(B+S) \leq \alpha(B)+\alpha(S)$, where $B+S=\{b+s: b \in B, s \in S\}$.

(g) $\alpha(\overline{\operatorname{conv}}(B))=\alpha(B)$. 
Recalling that the convex hull of a set $A \subseteq X$ is given by

$$
\operatorname{conv}(A)=\left\{\sum_{i=1}^{N} \alpha_{i} x_{i}: x_{i} \in A, \alpha_{i} \in \mathbb{R}, \alpha_{i} \geq 0, \sum_{i=1}^{N} \alpha_{i}=1\right\} .
$$

The details of $\alpha$ and its properties can be found in [5].

Definition 2.2. (see [6]). Assuming that $D \subset X$ the mapping $A: D \rightarrow X$ is said to be a condensing operator if $A$ is continuous, bounded (sends bounded sets into bounded sets), and for any nonrelatively compact and bounded set $S \subset D$,

$$
\alpha(A(S))<\alpha(S) .
$$

Remark 2.3. It is clear that a completely continuous operator is necessarily condensing.

The following lemmas are of great importance in the proof of our main results. The proofs can be found in [6].

In the following, we denote by $\alpha_{c}$ and $\alpha_{1}$ the noncompactness measure in $C$ and $C^{1}$, respectively.

Lemma 2.4. Let $S$ be a bounded subset of real numbers and $B$ a bounded subset of $X$. Then

$$
\alpha(S B)=\left(\sup _{t \in S}|t|\right) \alpha(B)
$$

where $S B=\{s b: s \in S, b \in B\}$.

Lemma 2.5. Let $A, B$ be bounded subsets of Banach spaces $X$ and $Y$ respectively with

$$
\|(x, y)\|=\max \{\|x\|,\|y\|\}
$$

Then

$$
\alpha(A \times B)=\max \{\alpha(A), \alpha(B)\} .
$$

Lemma 2.6. If $H$ is a bounded set in $C^{1}$, then

(ii) $\alpha_{1}(H) \geq \alpha(H(I))$.

(ii) $2 \alpha_{1}(H) \geq \alpha\left(H^{\prime}(I)\right)$.

Lemma 2.7. If $H$ is a bounded set in $C^{1}$ and $H^{\prime}$ equicontinuous, then 


$$
\alpha_{1}(H)=\max \left\{\max _{I} \alpha(H(t)), \max _{I} \alpha\left(H^{\prime}(t)\right)\right\} .
$$

The following lemma is an adaptation of a result of [1] to the case of a homeomorphism which is defined in $X$. We present here the demonstration for better understanding of the development of our research.

Lemma 2.8. For each $h \in C$, there exists a unique $Q_{\varphi}(h)=\int_{0}^{1} h(t) d t$ such that

$$
\int_{0}^{1} \varphi^{-1}\left(h(t)-Q_{\varphi}(h)\right) d t=0 .
$$

Moreover, the function $Q_{\varphi}: C \rightarrow X$ is continuous and sends bounded sets into bounded sets.

Proof. Let $h \in C$. We define the continuous application $G_{h}: X \longrightarrow X$ for

$$
G_{h}(a)=\int_{0}^{1} \varphi^{-1}(h(t)-a) d t .
$$

We now show that the equation

$$
G_{h}(a)=0
$$

has a unique solution $Q_{\varphi}(h)$. Let $a, b \in X$ be such that

$$
\int_{0}^{1} \varphi^{-1}(h(t)-a) d t=0, \int_{0}^{1} \varphi^{-1}(h(t)-b) d t=0,
$$

that is

$$
\int_{0}^{1} \varphi^{-1}(h(t)-a) d t=\int_{0}^{1} \varphi^{-1}(h(t)-b) d t .
$$

Using the fact that $\varphi^{-1}$ is a linear homeomorphism, we deduce the elementary equality $a=b$. Let us now show the existence. Because $\varphi^{-1}$ is linear, we obtain that

$$
\int_{0}^{1} \varphi^{-1}(h(t)-a) d t=\varphi^{-1}\left(\int_{0}^{1}(h(t)-a) d t\right) .
$$

Hence, if $a=\int_{0}^{1} h(t) d t$, then $G_{h}(a)=0$. Consequently for each $h \in C$, the equation

$$
G_{h}(a)=0
$$

has a unique solution. Thus, we define the function $Q_{\varphi}: C \longrightarrow X$ such that

$$
\int_{0}^{1} \varphi^{-1}\left(h(t)-Q_{\varphi}(h)\right) d t=0 .
$$

We now show that $Q_{\varphi}: C \longrightarrow X$ sends bounded sets into bounded sets. Let $A \subset C$ be a bounded set. Then, if $h \in A$, there exists $\rho>0$ such that

$$
\|h\|_{\infty} \leq \rho .
$$

On the other hand, as 


$$
Q_{\varphi}(A)=\left\{Q_{\varphi}(h): h \in A\right\} \text { and } Q_{\varphi}(h)=\int_{0}^{1} h(t) d t
$$

then $\left\|Q_{\varphi}(h)\right\| \leq \rho$. Therefore, $Q_{\varphi}$ sends bounded sets into bounded sets.

Finally, we show that $Q_{\varphi}$ is continuous on $C$. Let $\left(h_{n}\right)_{n} \subset C$ be a sequence such that $h_{n} \rightarrow h$ in $C$. Since the function $Q_{\varphi}$ sends bounded sets into bounded sets, then $\left(Q_{\varphi}\left(h_{n}\right)\right)_{n}$ is bounded. On the other hand, for all $n, m \in \mathbb{N}$, we have that

$$
\int_{0}^{1} \varphi^{-1}\left(h_{n}(t)-Q_{\varphi}\left(h_{n}\right)\right) d t=\int_{0}^{1} \varphi^{-1}\left(h_{m}(t)-Q_{\varphi}\left(h_{m}\right)\right) d t
$$

that provides

$$
\left\|\int_{0}^{1}\left(h_{n}(t)-h_{m}(t)\right) d t-\left(Q_{\varphi}\left(h_{n}\right)-Q_{\varphi}\left(h_{m}\right)\right)\right\|=0 .
$$

Hence,

$$
\left\|Q_{\varphi}\left(h_{n}\right)-Q_{\varphi}\left(h_{m}\right)\right\| \leq \int_{0}^{1}\left\|\left(h_{n}(t)-h_{m}(t)\right)\right\| d t .
$$

So, we obtain that $\left(Q_{\varphi}\left(h_{n}\right)\right)_{n}$ is a Cauchy sequence. Therefore, we can assume that

$$
\lim _{n \rightarrow \infty} Q_{\varphi}\left(h_{n}\right)=\widetilde{a}
$$

where for each $n \in \mathbb{N}$ we have that

$$
\lim _{n \rightarrow \infty} \int_{0}^{1} \varphi^{-1}\left(h_{n}(t)-Q_{\varphi}\left(h_{n}\right)\right) d t=0 .
$$

Using the dominated convergence theorem, we deduce that

$$
\int_{0}^{1} \varphi^{-1}(h(t)-\widetilde{a}) d t=0,
$$

so we have that $Q_{\varphi}(h)=\widetilde{a}$. Hence, $Q_{\varphi}$ is continuous.

\section{$3 \quad$ Fixed point formulations}

Let us consider the operator

$$
\begin{gathered}
M_{1}: C^{1} \rightarrow C^{1} \\
u \mapsto \varphi^{-1}\left(-Q_{\varphi}\left(H\left(N_{f}(u)\right)\right)\right)+H\left(\varphi^{-1}\left[H\left(N_{f}(u)\right)-Q_{\varphi}\left(H\left(N_{f}(u)\right)\right)\right]\right) .
\end{gathered}
$$

Here $\varphi^{-1}$ with an abuse of notation is understood as the operator $\varphi^{-1}: C \rightarrow C$ defined for $\varphi^{-1}(v)(t)=\varphi^{-1}(v(t))$. It is clear that $\varphi^{-1}$ is continuous and sends bounded sets into bounded sets.

In order to transform problem (1.1) to a fixed point problem we use Lemma 2.8.

Lemma 3.1. A map $u \in C^{1}$ is a solution of (1.1) if and only if $u$ is a fixed point of the operator $M_{1}$.

Proof. If $u \in C^{1}$ is solution of $(1.1)$, then 


$$
\left(\varphi\left(u^{\prime}(t)\right)\right)^{\prime}=N_{f}(u)(t)=f\left(t, u(t), u^{\prime}(t)\right), \quad u(0)=u(1), u(0)=u^{\prime}(0)
$$

for all $t \in[0,1]$. Applying $H$ to both members and using the fact that $u(0)=u^{\prime}(0)$, we deduce that

$$
\varphi\left(u^{\prime}(t)\right)=\varphi(u(0))+H\left(N_{f}(u)\right)(t) .
$$

By the inversion of $\varphi$, we have

$$
u^{\prime}(t)=\varphi^{-1}\left[H\left(N_{f}(u)\right)(t)+c\right],
$$

where $c=\varphi(u(0))$. Integrating from 0 to $t \in[0,1]$, we obtain

$$
u(t)=u(0)+H\left(\varphi^{-1}\left[H\left(N_{f}(u)\right)+c\right]\right)(t) .
$$

Because $u(0)=u(1)$, then

$$
\int_{0}^{1} \varphi^{-1}\left[H\left(N_{f}(u)\right)(t)+c\right] d t=0 .
$$

Using Lemma 2.8, it follows that $c=-Q_{\varphi}\left(H\left(N_{f}(u)\right)\right)$. Hence,

$$
u=\varphi^{-1}\left(-Q_{\varphi}\left(H\left(N_{f}(u)\right)\right)\right)+H\left(\varphi^{-1}\left[H\left(N_{f}(u)\right)-Q_{\varphi}\left(H\left(N_{f}(u)\right)\right)\right]\right) .
$$

Now suppose that $u \in C^{1}$ be such that $u=M_{1}(u)$. It follows that

$$
u(t)=\varphi^{-1}\left(-Q_{\varphi}\left(H\left(N_{f}(u)\right)\right)\right)+H\left(\varphi^{-1}\left[H\left(N_{f}(u)\right)-Q_{\varphi}\left(H\left(N_{f}(u)\right)\right)\right]\right)(t)
$$

for all $t \in[0,1]$. Since $\int_{0}^{1} \varphi^{-1}\left[H\left(N_{f}(u)\right)(t)-Q_{\varphi}\left(H\left(N_{f}(u)\right)\right)\right] d t=0$, therefore, we have that $u(0)=u(1)$. Differentiating (3.7), we obtain that

$$
\begin{aligned}
u^{\prime}(t) & =\varphi^{-1}\left[H\left(N_{f}(u)\right)-Q_{\varphi}\left(H\left(N_{f}(u)\right)\right)\right](t) \\
& =\varphi^{-1}\left[H\left(N_{f}(u)\right)(t)-Q_{\varphi}\left(H\left(N_{f}(u)\right)\right)\right] .
\end{aligned}
$$

Applying $\varphi$ to both of its members, and differentiating we have

$$
\left(\varphi\left(u^{\prime}(t)\right)\right)^{\prime}=N_{f}(u)(t), \quad u(0)=u(1), \quad u(0)=u^{\prime}(0)
$$

for all $t \in[0, T]$. This completes the proof.

In order to apply Leray-Schauder degree to the equivalent fixed point operator $M_{1}$, we introduced, for $\lambda \in[0,1]$, the family of boundary value problems

$$
\left\{\begin{array}{l}
\left(\varphi\left(u^{\prime}\right)\right)^{\prime}=\lambda f\left(t, u(t), u^{\prime}(t)\right. \\
u(0)=u(1)=u^{\prime}(0)
\end{array}\right.
$$


Notice that (3.8) coincide, for $\lambda=1$, with (1.1). For each $\lambda \in[0,1]$, the nonlinear operator associated to 3.8 for Lemma 3.1 is the operator $M(\lambda, \cdot)$, where $M$ is defined on $[0,1] \times C^{1}$ by

$$
M(\lambda, u)=\lambda \varphi^{-1}\left(-Q_{\varphi}\left(H\left(N_{f}(u)\right)\right)\right)+\lambda H\left(\varphi^{-1}\left[H\left(N_{f}(u)\right)-Q_{\varphi}\left(H\left(N_{f}(u)\right)\right)\right]\right) .
$$

Using the same arguments as in the proof of Lemma 3.1 we show that the system (3.8) is equivalent to the problem

$$
u=M(\lambda, u) .
$$

\section{Main results}

The main result of this paper is as follows.

Theorem 4.1. Let $X$ be a Banach space, and $\varphi^{-1}$ a linear homeomorphism with norm $k$. Assume that $f$ is continuous and satisfies the following conditions.

1. There exists a constant $c$ such that

$$
\|f(t, x, y)\| \leq c, \quad \text { for all } \quad(t, x, y) \in[0,1] \times X \times X .
$$

2. For all bounded subsets $A, B$ in $X$,

$$
\alpha(f([0,1] \times A \times B)) \leq k_{1} \max \{\alpha(A), \alpha(B)\}, \text { where } 0<k_{1}<1 / 6 k .
$$

Then problem (1.1) has at least one solution.

Proof. It is clear that $M_{1}$ maps bounded sets into bounded sets. The continuity of $M_{1}$ follows from the continuity of the operators $N_{f}$ and $H$. Finally, we show that $M_{1}$ is a condensing operator ( $\alpha$-condensing). In fact, for a bounded set $\Lambda$ in $C^{1}$, there exists a constant $L_{1}>0$ such that

$$
\left\|N_{f}(u)\right\|_{\infty} \leq L_{1} \text {, for all } u \in \Lambda .
$$

For $t, t_{1} \in[0,1]$ we have that

$$
\begin{aligned}
\left\|\left(M_{1} u\right)^{\prime}(t)-\left(M_{1} u\right)^{\prime}\left(t_{1}\right)\right\| & \leq k\left\|H\left(N_{f}(u)\right)(t)-H\left(N_{f}(u)\right)\left(t_{1}\right)\right\| \\
& \leq k\left\|\int_{t_{1}}^{t} f\left(s, u(s), u^{\prime}(s)\right) d s\right\| \\
& \leq k L_{1}\left|t-t_{1}\right| .
\end{aligned}
$$

So, $\left(M_{1} \Lambda\right)^{\prime}$ is equicontinuous. Applying Lemma 2.7 there exists $\tau \in[0,1]$ or $\omega \in[0,1]$ with 


$$
\alpha_{1}\left(M_{1} \Lambda\right)=\alpha\left(\left(M_{1} \Lambda\right)(\tau)\right)
$$

or

$$
\alpha_{1}\left(M_{1} \Lambda\right)=\alpha\left(\left(M_{1} \Lambda\right)^{\prime}(\omega)\right) .
$$

Let us consider the first case.

$$
\begin{aligned}
\alpha_{1}\left(M_{1} \Lambda\right) & =\alpha\left(\left(M_{1} \Lambda\right)(\tau)\right) \\
& =\alpha\left(\left\{\left(M_{1} u\right)(\tau): u \in \Lambda\right\}\right),
\end{aligned}
$$

where $\left(M_{1} u\right)(\tau)=\varphi^{-1}\left(-Q_{\varphi}\left(H\left(N_{f}(u)\right)\right)\right)+H\left(\varphi^{-1}\left[H\left(N_{f}(u)\right)-Q_{\varphi}\left(H\left(N_{f}(u)\right)\right)\right]\right)(\tau)$.

Using the properties of the noncompactness measure $\alpha$, we obtain

$$
\begin{aligned}
\alpha_{1}\left(M_{1} \Lambda\right) & \leq \alpha\left(\left\{\varphi^{-1}\left(-Q_{\varphi}\left(H\left(N_{f}(u)\right)\right)\right): u \in \Lambda\right\}\right) \\
& +\alpha\left(\left\{H\left(\varphi^{-1}\left[H\left(N_{f}(u)\right)-Q_{\varphi}\left(H\left(N_{f}(u)\right)\right)\right]\right)(\tau): u \in \Lambda\right\}\right) .
\end{aligned}
$$

Using the fact that $\varphi^{-1}$ is a linear homeomorphism with norm $k, Q_{\varphi}\left(H\left(N_{f}(u)\right)\right)=$ $\int_{0}^{1} H\left(N_{f}(u)\right)(t) d t$, and Lemma 2.4, we deduce that

$$
\begin{aligned}
\alpha\left(\left\{\varphi^{-1}\left(-Q_{\varphi}\left(H\left(N_{f}(u)\right)\right)\right): u \in \Lambda\right\}\right) & \leq k \alpha\left(\overline{\operatorname{conv}}\left\{H\left(N_{f}(u)\right)(s): s \in[0,1], u \in \Lambda\right\}\right) \\
& \leq k \alpha\left(\left\{\int_{0}^{s} f\left(t, u(t), u^{\prime}(t)\right) d t: s \in[0,1], u \in \Lambda\right\}\right) \\
& \leq k \alpha\left([0,1] \overline{\operatorname{conv}}\left\{f\left(t, u(t), u^{\prime}(t)\right): t \in[0,1], u \in \Lambda\right\}\right) \\
& \leq k \alpha\left(\left\{f\left(t, u(t), u^{\prime}(t)\right): t \in[0,1], u \in \Lambda\right\}\right) .
\end{aligned}
$$

On the other hand, we have

$$
\begin{array}{r}
\alpha\left(\left\{H\left(\varphi^{-1}\left[H\left(N_{f}(u)\right)-Q_{\varphi}\left(H\left(N_{f}(u)\right)\right)\right]\right)(\tau): u \in \Lambda\right\}\right) \\
\leq \alpha\left(\left\{\int_{0}^{\tau} \varphi^{-1}\left[H\left(N_{f}(u)\right)(s)-Q_{\varphi}\left(H\left(N_{f}(u)\right)\right)\right] d s: u \in \Lambda\right\}\right) \\
\leq k \alpha\left(\left\{H\left(N_{f}(u)\right)(s): s \in[0,1], u \in \Lambda\right\}\right)+\alpha\left(\left\{\varphi^{-1}\left(-Q_{\varphi}\left(H\left(N_{f}(u)\right)\right)\right): u \in \Lambda\right\}\right) \\
\leq 2 k \alpha\left(\left\{f\left(t, u(t), u^{\prime}(t)\right): t \in[0,1], u \in \Lambda\right\}\right) .
\end{array}
$$

Hence,

$$
\begin{aligned}
\alpha_{1}\left(M_{1} \Lambda\right) & \leq 3 k \alpha\left(\left\{f\left(t, u(t), u^{\prime}(t)\right): t \in[0,1], u \in \Lambda\right\}\right) \\
& \leq 3 k \alpha\left(f\left([0,1] \times \Lambda([0,1]) \times \Lambda^{\prime}([0,1])\right)\right) .
\end{aligned}
$$

Using the assumption 2, we have that

$$
\alpha_{1}\left(M_{1} \Lambda\right) \leq 3 k k_{1} \max \left\{\alpha(\Lambda([0,1])), \alpha\left(\Lambda^{\prime}([0,1])\right)\right\} .
$$


This implies, by Lemma 2.6

$$
\alpha_{1}\left(M_{1} \Lambda\right) \leq 6 k k_{1} \alpha_{1}(\Lambda) .
$$

Consider the alternative case. Proceeding as before, we obtain

$$
\alpha_{1}\left(M_{1} \Lambda\right)=\alpha\left(\left(M_{1} \Lambda\right)^{\prime}(\omega)\right) \leq 6 k k_{1} \alpha_{1}(\Lambda) .
$$

Therefore, in either case, we obtain

$$
\alpha_{1}\left(M_{1} \Lambda\right) \leq 6 k k_{1} \alpha_{1}(\Lambda) .
$$

By the assumption 2, we get $0<6 k k_{1}<1$, therefore $M_{1}$ is $\alpha$-condensing.

On the other hand, let $(\lambda, u) \in[0,1] \times C^{1}$ be such that $u=M(\lambda, u)$. Using (3.10) we have that $u$ is solution of (3.8), which implies that

$$
u^{\prime}=\lambda \varphi^{-1}\left[H\left(N_{f}(u)\right)-Q_{\varphi}\left(H\left(N_{f}(u)\right)\right)\right], u(0)=u(1)=u^{\prime}(0) .
$$

Using the fact that $\varphi^{-1}$ is a linear homeomorphism with norm $k$ and $Q_{\varphi}\left(H\left(N_{f}(u)\right)\right)=$ $\int_{0}^{1} H\left(N_{f}(u)\right)(t) d t$, we deduce that

$$
\left\|u^{\prime}(t)\right\| \leq 2 k c \quad(t \in[0,1]) .
$$

Hence, $\left\|u^{\prime}\right\|_{\infty} \leq 2 k c:=\beta$. Because $u \in C^{1}$ is such that $u^{\prime}(0)=u(0)$ we have that

$$
\|u(t)\| \leq\|u(0)\|+\int_{0}^{t}\left\|u^{\prime}(s)\right\| d s \leq\|u(0)\|+\int_{0}^{1}\left\|u^{\prime}(s)\right\| d s \leq 2 \beta \quad(t \in[0,1]),
$$

and hence

$$
\|u\|_{1} \leq 2 \beta .
$$

Finally, we show the existence of at least one solution of (1.1) using the homotopy invariance of the degree for $\alpha$-condensing maps. Let $B$ be bounded in $C^{1}$. Then

$$
\begin{aligned}
\alpha_{1}(M([0,1] \times B)) & =\alpha_{1}(M(\lambda, u): \lambda \in[0,1], u \in B) \\
& \leq 6 k k_{1} \alpha_{1}(B) .
\end{aligned}
$$

Then we have that for each $\lambda \in[0,1]$, the degree $\operatorname{deg}_{N}\left(I-M(\lambda, \cdot), B_{r}(0), 0\right)$ is well defined for any $r>2 \beta$ and, by the properties of that degree, that

$$
\operatorname{deg}_{N}\left(I-M(1, \cdot), B_{r}(0), 0\right)=\operatorname{deg}_{N}\left(I-M(0, \cdot), B_{r}(0), 0\right)=1 .
$$

Then, from the existence property of degree, there exists $u \in B_{r}(0)$ such that $u=$ $M(1, u)=M_{1}(u)=u$, which is a solution for (1.1).

Remark 4.2. In [12], there are Dirichlet boundary conditions, our result is for a more general type of boundary conditions. 
By Theorem 4.1, we can immediately get the following corollary about the existence of a solution of the problem (1.1).

Corollary 4.3. Let $X$ be a Banach space, and $\varphi^{-1}$ a linear homeomorphism with norm $k$. Suppose that $f$ is completely continuous and that there exists one number $c \geq 0$ such that

$$
\|f(t, x, y)\| \leq c, \quad \text { for all } \quad(t, x, y) \in[0,1] \times X \times X .
$$

Then problem (1.1) has at least one solution.

Now we consider an example to illustrate our results.

Example 4.4. Consider the boundary value problem in

$$
X=l^{\infty}=\left\{x=\left(x_{1}, x_{2}, \ldots, x_{i}, \ldots\right): \sup _{i}\left|x_{i}\right|<\infty\right\},
$$

with norm $\|x\|=\sup _{i}\left|x_{i}\right|$.

$$
\begin{gathered}
u_{i}^{\prime \prime}=\frac{\cos \left(u_{i}\right)}{i^{2}} \quad t \in[0,1], \\
u_{i}(0)=u_{i}(1)=u_{i}^{\prime}(0) .
\end{gathered}
$$

Now we can regarded this problem as a problem of the form (1.1), where $u=$ $\left(u_{1}, u_{2}, \ldots, u_{i}, \ldots\right) \in l^{\infty}, f=\left(f_{1}, f_{2}, \ldots, f_{i}, \ldots\right)$ with

$$
f_{i}(t, x, y)=\frac{\cos \left(x_{i}\right)}{i^{2}} \quad(t, x, y) \in[0,1] \times l^{\infty} \times l^{\infty} .
$$

It is clear that $f:[0,1] \times l^{\infty} \times l^{\infty} \rightarrow l^{\infty}$ is a continuous function and that

$$
\begin{aligned}
\|f(t, x, y)\| & =\sup _{i}\left|f_{i}(t, x, y)\right| \\
& \leq 1 .
\end{aligned}
$$

On the other hand, for any bounded subsets $A, B \subset l^{\infty}$ we have

$$
\alpha(f([0,1] \times A \times B))=0,
$$

and hence

$$
\alpha(f([0,1] \times A \times B)) \leq \frac{1}{7} \max \{\alpha(A), \alpha(B)\} .
$$

So, by Theorem 4.1 we get one solution. 


\section{Acknowledgements}

This research was supported by CAPES and CNPq/Brazil.

\section{References}

[1] C. Bereanu and J. Mawhin, Boundary-value problems with non-surjective $\varphi$ laplacian and one-sided bounded nonlinearity, Advances Differential Equations. 11 (2006), 35-60.

[2] V. Bouches and J. Mawhin, Boundary value problems for a class of first order quasilinear ordinary differential equations, Portugal. Math. (N.S). 71 (2014), 217-247.

[3] J. Chandra, V. Lakshmikantham, and A. R. Mitchell, Existence of solutions of boundary value problems for nonlinear second-order systems in a Banach space, Nonlinear Analysis. 2 (1978), 157-168.

[4] H. Chen and P. Li, Three-point boundary value problems for second-order ordinary differential equations in Banach spaces, Computers and Mathematics with Applications. 56 (2008), 1852-1860.

[5] K. Deimling, Nonlinear Functional Analysis, Springer, Berlin, 1985.

[6] D. Guo, Y. Je Cho and J. Zhu, Partial ordering metods in nonlinear problems, Hauppauge, New York, 2004.

[7] D. J. Guo and V. Lakshmikantham, Nonlinear Problems in Abstract cones, vol. 5 of Notes and Reports in Mathematics in Science and Engineering, Academic Press, San Diego, Calif, 1988.

[8] R. Manásevich and J. Mawhin, Periodic solutions for nonlinear systems with p-laplacian-like operators, Differential Equations. 145 (1997), 367-393.

[9] H. Mönch, Boundary value problems for nonlinear ordinary differential equations of second order in Banach spaces, Nonlinear Analysis. 4 (1980), 985-999.

[10] D. P. D. Santos, Problems with mixed boundary conditions in Banach spaces, Chinese Journal of Mathematics. Article ID 7838102(2017). doi:10.1155/2017/7838102.

[11] S. Szufla, Boundary value problems for nonlinear ordinary differential equations of second order in Banach spaces, Nonlinear Analysis. 8 (1984), 1481-1487. 
[12] Zhou, WX, Peng, j, Existence of solution to a second-order boundary value problem via noncompactness measures, Discrete Dynamics in Nature and Society. Article ID 786404(2012). doi:10.1155/2012/786404. 